\title{
ON CALCULATION OF THE WITTEN INVARIANTS OF 3-MANIFOLDS
}

\author{
EUGENE RAFIKOV, DUŠAN REPOVŠ and FULVIA SPAGGIARI
}

(Received 31 July 2001; revised 13 November 2002)

Communicated by S. Gadde

\begin{abstract}
In this paper we present a short definition of the Witten invariants of 3-manifolds. We also give simple proofs of invariance of those obtained for $r=3$ and $r=4$. Our definition is extracted from the 1993 paper of Lickorish and the Prasolov-Sossinsky book, where it is dispersed over 20 pages. We show by several examples that it is indeed convenient for calculations.
\end{abstract}

2000 Mathematics subject classification: primary 57M25; secondary 57N10.

Keywords and phrases: Witten invariant, Kauffman bracket, plane diagram, Dehn surgery, 3-manifold.

\section{Definition of the Witten invariant}

The construction of Witten invariants of 3-manifolds and the proof of their invariance use deep ideas from the quantum field theory and the theory of Temperley-Lieb algebras and are not short. But a mathematician might want to calculate and apply these invariants without necessarily understanding their origin. The definition of the Witten invariants in [6, page 660] is direct and short, but is not so convenient for calculations. In this paper we present a short definition of the Witten invariants (Theorem 1.3) which is extracted from [8] (where it is dispersed over 20 pages, mixed with the proof of invariance) and we show by several examples that it is indeed more convenient for calculations. In Section 2 we give a new simple proof of invariance for $r=4$.

Repovš was supported in part by the Ministry for Science and Technology of the Republic of Slovenia research grant No. J1-0885-0101-98. Spaggiari was partially supported by the GNSAGA of the CNR (National Research Council) of Italy, by the Ministero per la Ricerca Scientifica e Tecnologica of Italy within the project 'Proprietà Geometriche delle Varietà Reali e Complesse' and by a research grant of the University of Modena and Reggio Emilia.

(c) 2003 Australian Mathematical Society 1446-7887/03 \$A2.00+0.00 


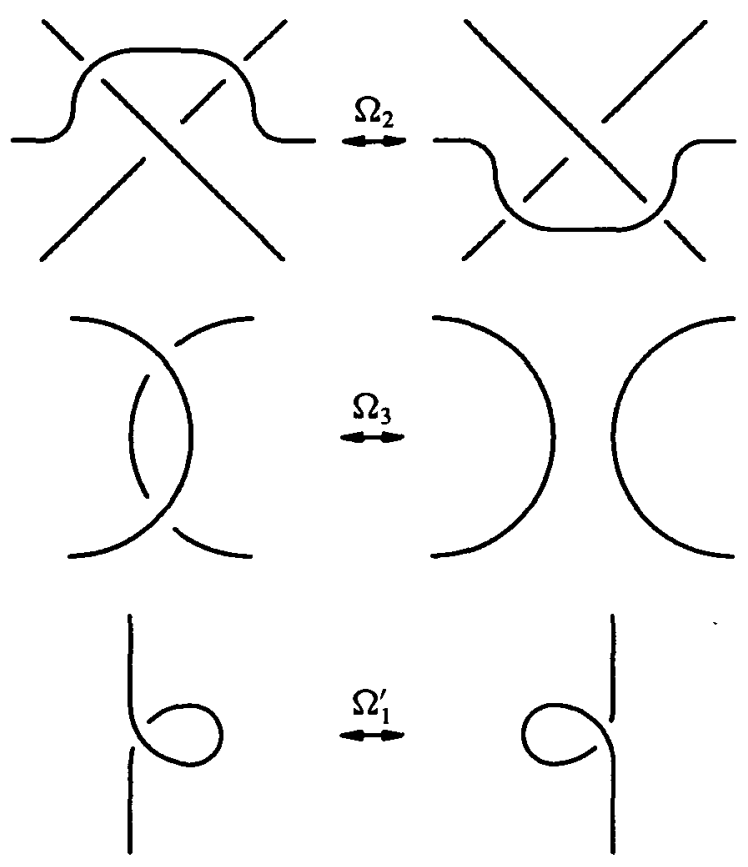

FIGURE 1 .

The definition of the Witten invariant is based on the representation of 3-manifolds by (unoriented) plane diagrams. By a plane diagram we understand a set of circles in $\mathbb{R}^{2}$ in general position, with chosen undercrossing and overcrossing at each intersection point. For every single component $D_{k}$ of the plane diagram $D$ we can determine its integer framing as follows. Choose any orientation of $D_{k}$. Define the framing as the sum of the signs $( \pm 1)$ of all of its crossings. Note that this number is independent of the choice of orientation on $D_{k}$.

Suppose that $L$ is an unoriented link in $S^{3}$ and that an integer $g(k)$ is assigned to each component $L_{k}$ of $L$. Then the pair $(L, g)$ is called a framed link. We say that a framed link $(L, g)$ is represented by a plane diagram $D$, if $D$ is a diagram for $L$ in the usual sense and $g(k)$ equals the framing of $D_{k}$, for every single component $D_{k}$ of $D$.

It is well known that every closed oriented 3-manifold can be obtained from the 3-sphere $S^{3}$ by the Dehn surgery on some framed link $(L, g)$. Denote by $\chi_{D}$ the 3-manifold obtained by the Dehn surgery along the framed unoriented link, corresponding to $\dot{D}$.

Proposition $1.1([1,3])$. Suppose that $D$ and $D^{\prime}$ are plane diagrams. Then $\chi_{D} \cong$ $\chi_{D^{\prime}}$ if and only if $D^{\prime}$ can be obtained from $D$ by a sequence of the Reidemeister moves $\Omega_{1}^{\prime}, \Omega_{2}$, and $\Omega_{3}$ shown in Figure 1 and the Fenn-Rourke moves shown in Figures 4 (a)-(b). 

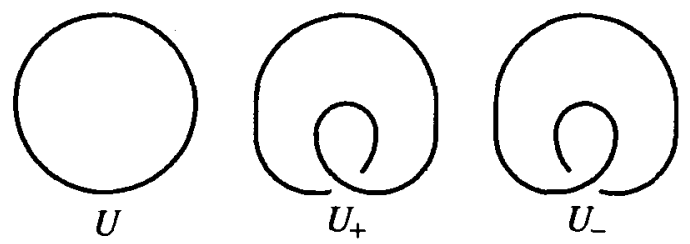

(a)
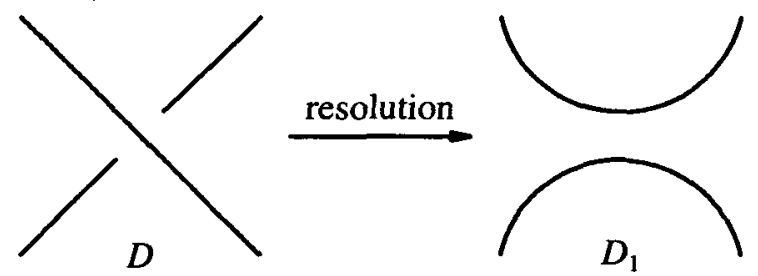

(b)
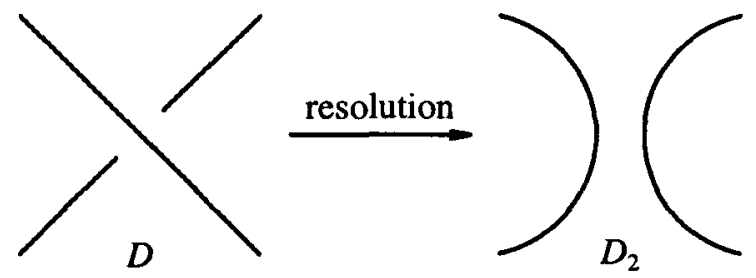

(c)

FIGURE 2.

For a plane diagram $D=\left(D_{1}, \ldots, D_{n}\right)$, consider any oriented link $L=\left(L_{1}, \ldots, L_{n}\right)$ in $S^{3}$, whose plane projection coincides with $D$. Let $b_{p q}=\operatorname{lk}\left(L_{p}, L_{q}\right)$ for $p \neq q$ and let $b_{k k}$ equal the framing of $D_{k}$. Denote by $b_{+}(D)$ and $b_{-}(D)$ the numbers of positive and negative eigenvalues of the linking coefficients matrix $\left(b_{p q}\right)$ of $L$. Let $\sigma(D)=b_{+}(D)-b_{-}(D)$ be the signature of $\left(b_{p q}\right)$ and $D \cdot D=\sum_{p, q} b_{p q}(\bmod 4)$. Clearly, the above numbers depend only on $D$, not on $L$ and its orientation. We set $\sigma=\sigma(D)$ and $b_{ \pm}=b_{ \pm}(D)$ when $D$ is fixed and no confusion can arise. Let $|D|$ be the number of components in $D$. Then $\operatorname{rk} H_{1}\left(\chi_{D}, \mathbb{Z}\right)=|D|-b_{+}(D)-b_{-}(D)$. Denote by $\# D$ the number of crossings in $D$. Let $|D|_{+}$and $|D|_{-}$be the numbers of the connected components after resolution of all the crossings as shown in Figures 2 (b) and $(c)$, respectively.

In what follows capital Latin letters denote (unoriented) plane diagrams (in [8] they are sometimes called framed diagrams). Let $U_{+}, U$ and $U_{-}$be the diagrams representing the unknot with framings $+1,0$ and -1 , respectively (see Figure 2 (a)).

Everywhere below we suppose that diagrams in the equalities coincide except where shown in corresponding figures.

The Kauffman bracket is a function $(\cdot):$ \{plane diagrams\} $\rightarrow \mathbb{Z}\left[a^{ \pm 1}\right]$, defined by 
the following three properties (see for example [8, Section $26,(1)-(3)]$ ):

(a) $\langle D\rangle=a\left\langle D_{1}\right\rangle+a^{-1}\left\langle D_{2}\right\rangle$, where the diagrams $D, D_{1}$ and $D_{2}$ are shown in Figures 2 (b)-(c);

(b) $\langle D \cup U\rangle=\left(-a^{2}-a^{-2}\right)\langle D\rangle$; and

(c) $\langle\emptyset\rangle=1$.

The normalization of (c) is not entirely standard, but in this paper it is more convenient to use $\langle D\rangle$ instead of the original Kauffman bracket $\langle D\rangle /\left(-a^{2}-a^{-2}\right)$.

PROPOSITION 1.2 ([2, 5, 8, Section 26]). The Kauffman bracket is unchanged by the Reidemeister moves $\Omega_{1}^{\prime}, \Omega_{2}$, and $\Omega_{3}$.

THEOREM $1.3([7,8,27.3,28.2] \mathrm{cf}$. [6]). Fix integers $r \geq 3$ and $k=1, \ldots, 4 r-1$ relatively prime to $2 r$. Define the polynomial

$$
\omega(\alpha)=\prod_{\substack{l=1 \\ k \pm l \neq r, 3 r}}^{r-1}\left(\alpha-2 \cos \frac{\pi l}{r}\right) .
$$

For a plane diagram $D$ with $n=|D|$ components, let $D^{\left(k_{1}, \ldots, k_{n}\right)}$ be the diagram obtained from $D$ by taking $k_{i}$ curves, close and parallel to the $i$-th component. Define $a$ polylinear map $f_{D}:(\mathbb{C}[\alpha])^{n} \rightarrow \mathbb{C}$ on the basic elements by setting $f_{D}\left(\alpha^{k_{1}}, \ldots, \alpha^{k_{n}}\right)=$ $\left\langle D^{\left(k_{1}, \ldots, k_{n}\right)}\right)$ at $a=\exp (\pi i k / 2 r)$. Then the following number (the Witten invariant for $r$ at a) depends only on the oriented $\chi_{D}$ :

$$
W(D)=f_{U_{+}}^{-b_{+}(D)}(\omega) \cdot f_{U_{-}}^{-b_{-}(D)}(\omega) \cdot f_{D}(\omega, \ldots, \omega)
$$

REMARK 1.4. It follows from [Lic93, Lemma 4] or [PrSo97, Proposition 29.4] that $f_{U_{ \pm}}(\omega) \neq 0$. For $r=3$ and $r=4$, we easily verify it below.

REMARK 1.5. It is easier to calculate the polynomial $\omega$ not by the explicit formula of Theorem 1.3 but by the following algorithm. Define the (renormalized Chebyshev) polynomials $S_{n}(\alpha)$ by the recurrence formula $S_{0}=1, S_{1}=\alpha$ and $S_{n+1}=\alpha S_{n}-S_{n-1}$. Then

$$
\omega=(-1)^{r-k+1} \sum_{n=0}^{r-2}(-1)^{n} \frac{\sin (\pi k(n+1) / r)}{\sin (\pi k / r)} S_{n} .
$$

Indeed, it suffices to show that the above sum has exactly $r-2$ roots $2 \cos (\pi l / r)$, where $1 \leq l \leq r-1$ and $k \pm l \neq r, 3 r$ (there are exactly $r-2$ numbers $l$ with these 
properties). Note that $\sin x \cdot S_{n}(2 \cos x)=\sin (n+1) x$. Then

$$
\begin{aligned}
& 2 \sin (\pi k / r) \sin (\pi l / r) \omega(2 \cos (\pi l / r)) \\
& \quad=2 \sum_{n=0}^{r-2}(-1)^{n} \sin (\pi k(n+1) / r) \sin (\pi l(n+1) / r) \\
& \quad=\sum_{n=1}^{r-1}(-1)^{n+1} \cos (\pi(k+l) n / r)-\sum_{n=1}^{r-1}(-1)^{n+1} \cos (\pi(k-l) n / r) \\
& =(-1)^{r+k+l}-(-1)^{r+k-l}=0 .
\end{aligned}
$$

REMARK 1.6. For odd $r$ in Theorem 1.3, one can also take $k=1, \ldots, 2 r-1$ relatively prime to $2 r, a=e^{\pi i k / r}$ and

$$
\omega(\alpha)=\prod_{\substack{l=1 \\ 2 k \pm l \neq r, 3 r}}^{r-1}\left(\alpha-2 \cos \frac{\pi l}{r}\right)
$$

EXAMPLE 1.7. $W\left(S^{3}\right)=W\left(U_{ \pm}\right)=1$.

EXAMPLE 1.8. It follows from $[9,3.4]$ that a changing of the orientation of 3manifold has the effect of complex conjugation on the Witten invariants.

EXAMPLE 1.9. For $a=e^{\pi i / 3}$, we have $\langle D\rangle=1$. This can be verified by induction on the number of crossings in $D$ using the definition of the Kauffman bracket.

EXAMPLE 1.10. Suppose $r=3, k=1$ and $a=e^{\pi i / 3}$. Then $\omega=1+\alpha$ (see Remark 1.5) and by Example 1.9, $\langle D\rangle=1$. Hence

$$
W(D)=2^{-b_{+}} \cdot 2^{-b_{-}} \sum_{P \subset D} 1=2^{|D|-b_{+}-b_{-}}=2^{\text {rk } H_{1}\left(\chi_{D}\right)} .
$$

REMARK 1.11. Observe that if $\omega$ is replaced in Theorem 1.3 throughout by $\mu \omega$, where $\mu$ is a constant complex number, then another invariant is obtained. The new invariant is the old one multiplied by $\mu^{\mathrm{rk} H_{1}\left(\chi_{D}, \mathbb{Z}\right)}$. Choose $\mu \in \mathbb{C}$ so that $\mu^{-2}=$ $f_{U_{+}}(\omega) \cdot f_{U_{-}}(\omega)$. This means that $f_{U_{+}}(\mu \omega)^{-1}=f_{U_{-}}(\mu \omega)$. So we obtain the Witten invariant $R(D)=f_{D}(\mu \omega, \mu \omega, \ldots, \mu \omega) f_{U_{-}}(\mu \omega)^{\sigma}$.

LEMMA 1.12. For the Kauffman bracket at $a=e^{\pi i / 6}$, we have

$$
\langle D\rangle=(-1)^{|D|_{+}} \cdot i^{\# D}=(-1)^{|D|_{-}} \cdot i^{-\# D}=i^{2|D|-D \cdot D} .
$$


Proof. First we prove that

$$
\langle D\rangle=i\left\langle D_{1}\right\rangle=-i\left\langle D_{2}\right\rangle,
$$

where the diagrams $D, D_{1}$ and $D_{2}$ differ as shown in Figures 2 (b)-(c). This can be verified by induction on $\# D$. It follows from (a) that we must only prove that $\left\langle D_{1}\right\rangle=-\left\langle D_{2}\right\rangle$. The base $\# D=0,1$ is easy. If $\# D \geq 2$, then $D_{1}$ and $D_{2}$ have a crossing point. The induction hypothesis then gives $\left\langle D_{1}\right\rangle=i\left\langle D_{11}\right\rangle=-i\left\langle D_{21}\right\rangle=-\left\langle D_{2}\right\rangle$, where the diagrams $D_{i}$ and $D_{i 1}$ are identical except where shown in Figure 2 (b) $(i=1,2)$ and $(*)$ is proved. From this at once we obtain the first two equalities of Lemma 1.12 .

Now we prove that $(D\rangle=i^{2|D|-D \cdot D}$. The equality is evident for trivial diagrams $D$ (that is, for diagrams without any crossings). By Proposition 1.2 it also holds for diagrams of the unoriented trivial link. There exists an orientation $\bar{D}=\left(\bar{D}_{1}, \ldots, \bar{D}_{k}\right)$ of $D$ such that $b_{p q}$ equals the sum of the signs \pm 1 of all the crossings where $\bar{D}_{p}$ overcrosses $\bar{D}_{q}$. It is well known that $D$ can be obtained from the diagram of the trivial link by changing some overcrossings by undercrossings and reverse operations. Clearly, $i^{-D \cdot D}$ is multiplied by -1 under such operation. It follows from $(*)$ that $\langle D\rangle$ is also multiplied by -1 and we are done.

EXAMPLE 1.13. Suppose $r=3, k=1$ and $a=e^{\pi i / 6}$. Then $\omega=1-\alpha, f_{U_{+}}(\omega)=$ $1-i, f_{U_{-}}(\omega)=1+i, \mu=1 / \sqrt{2}$ and $f_{U_{-}}(\mu \omega)=e^{\pi i / 4}$. Hence, by Lemma 1.12 the Witten invariant of Remark 1.11 equals

$$
R(D)=2^{-|D| / 2} e^{\pi i \sigma / 4} \sum_{P \subset D}(-1)^{|P|}\langle P\rangle=2^{-|D| / 2} e^{\pi i \sigma / 4} \sum_{P \subset D} i^{-P . P} .
$$

Note that $R(D)$ is obtained from $\tau_{3}(D)$ of [4, page 521] by complex conjugation.

EXAMPLE 1.14. Let $r=4, k=1$ and $a=e^{\pi i / 8}$. We have $\omega=\alpha^{2}-\sqrt{2} \alpha$, $\left\langle U_{+}^{2}\right\rangle=\left\langle U_{-}^{2}\right\rangle=0, f_{U_{+}}(\omega)=-2 e^{3 \pi i / 8}, f_{U_{-}}(\omega)=2 e^{5 \pi i / 8}$ and $\mu=1 / 2$. Therefore, the Witten invariant from Remark 1.11 equals

$$
R(D)=(-1)^{|D|} 2^{-|D| / 2} e^{5 \pi i \sigma / 8} \sum_{P \subset D}(-\sqrt{2})^{-|P|}\langle D \circ P\rangle,
$$

where $D \circ P$ is the diagram obtained from $D$ by drawing circles, parallel and close to the components of $P$, see for example [4, Section 6].

\section{Simple proofs of Theorem 1.3 for $r=3$ and $r=4$}

We only consider the case $k=1$. The case of arbitrary $k$ (for given $r$ ) is proved analogously. 


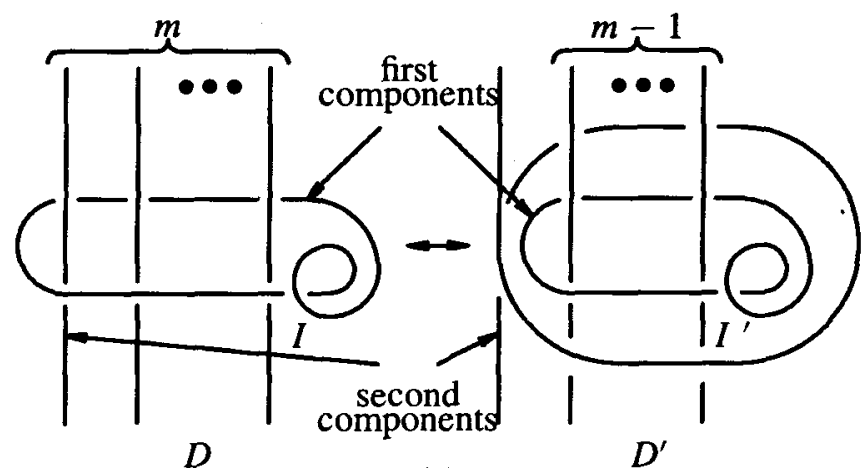

(a)

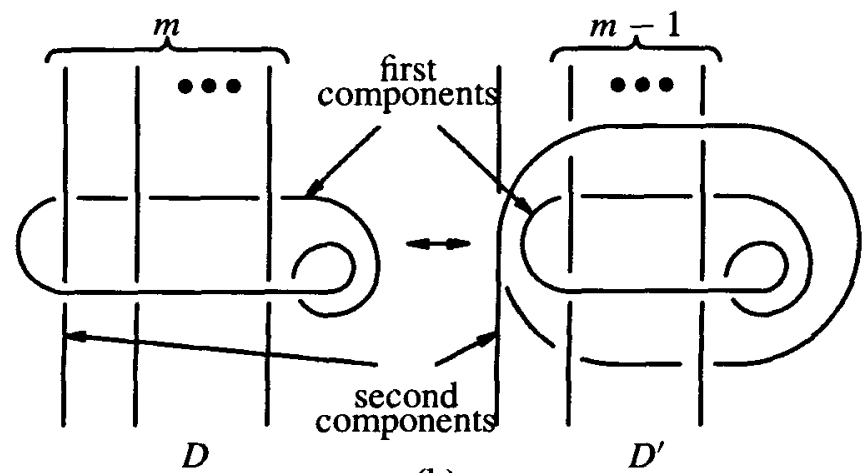

(b)

FIGURE 3.

LEMMA 2.1. The numbers $b_{+}(D)$ and $b_{-}(D)$ remain unchanged under the moves in Figures 3 (a)-(b).

PROOF. Let $D$ and $D^{\prime}$ be the diagrams shown in Figures 3 (a)-(b). It is easy to see that $\left(b_{p q}\right)=\left(x_{p q}\right)^{t}\left(b_{p q}^{\prime}\right)\left(x_{p q}\right)$ for $x_{p p}=1, x_{12}= \pm 1$ and $x_{p q}=0$ otherwise, where the first two components of $D$ and $D^{\prime}$ are specified. Hence the lemma follows.

It follows from Proposition 1.1 and Proposition 1.2 that for proving the invariance of $W(D)$ one need only verify the invariance under the Fenn-Rourke moves in Figure 4 (a)-(b).

PROOF OF THEOREM 1.3 FOR $r=3$ AND $k=1$. Let $a=e^{\pi i / 6}$. The proof is essentially the same as in [4, page 521], where invariance under the Kirby transformations was verified. It follows from Lemma 1.12 and Example 1.13 that

$$
R(D)=2^{-|D| / 2} e^{\pi i \sigma / 4} \sum_{P \subset D}(-1)^{|P|+|P|+} i^{* D}=2^{-|D| / 2} e^{\pi i \sigma / 4} \sum_{P \subset D}(-1)^{|P|+|P|-} i^{-\# D} .
$$




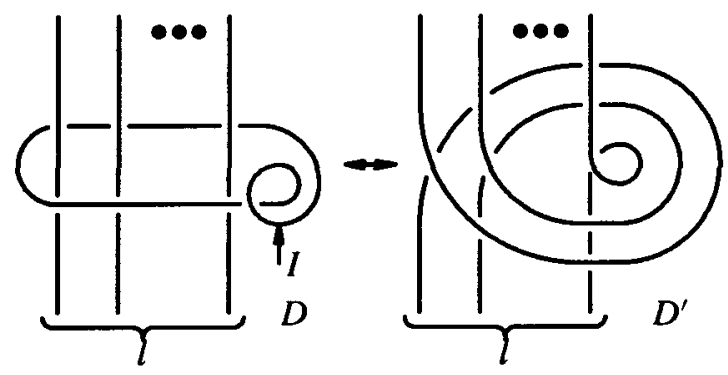

(a)

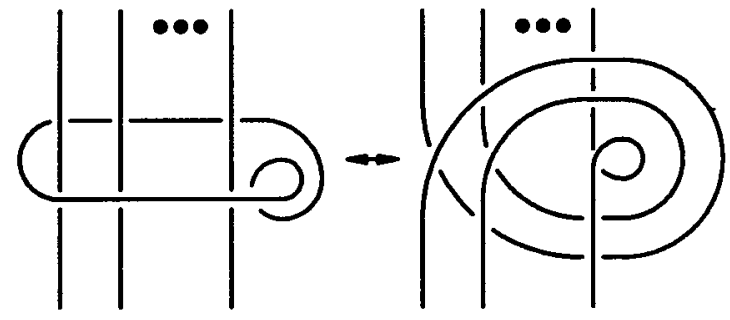

(b)

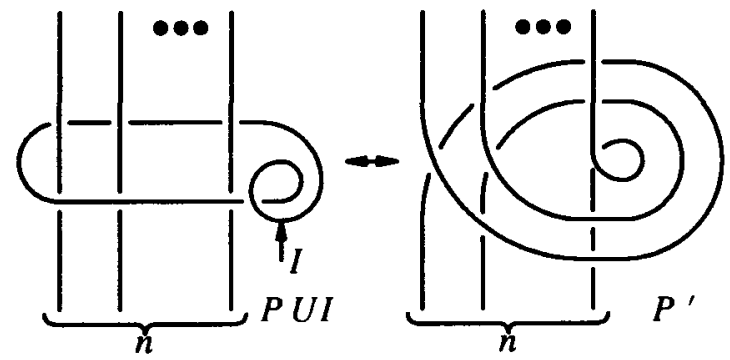

(c)
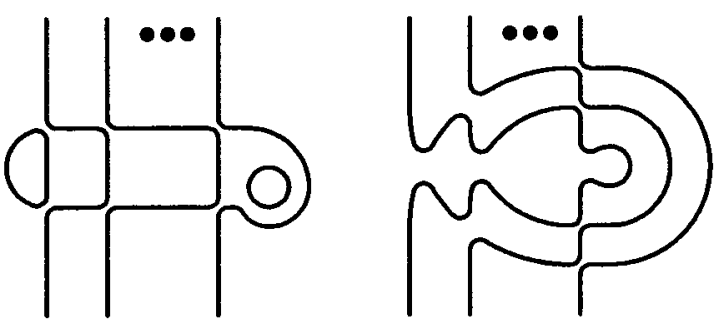

(d)

FIGURE 4. 
We prove the invariance under the move in Figure 4 (a) using the formula for $R(D)$ involving $|\cdot|_{+}$. The invariance under the move in Figure $4(\mathrm{~b})$ is verified analogously using the formula for $R(D)$ involving $|\cdot|_{-}$. Denote by $D, D^{\prime}$, and $I$ the diagrams shown in Figure 4 (a). Clearly, the Fenn-Rourke move in Figure 4 (a) is decomposed into $l$ second Kirby moves in Figure 3 (a) (for $m=l, \ldots, 1$ ) and one first Kirby move in Figure 5. Since $\sigma\left(D^{\prime} \cup U_{+}\right)=\sigma\left(D^{\prime}\right)+1$, it follows from Lemma 2.1 that $\sigma(D)=\sigma\left(D^{\prime}\right)+1$. Let $P$ denote an arbitrary subdiagram of $D \backslash I$. Clearly, $|P \cup I|=|P|+1$ and $|P \cup I|_{+}=|P|+2$. Hence, we have

$$
\begin{aligned}
R(D) & =\frac{2^{-\left|D^{\prime}\right| / 2} e^{\pi i \sigma\left(D^{\prime}\right) / 4}}{1-i} \sum_{P \subset D \backslash I}\left((-1)^{|P|+|P|_{+}} i^{\# P}+(-1)^{|P \cup I|+|P \cup I|_{+}} i^{\#(P \cup I)}\right) \\
& =2^{-\mid D^{\prime} / / 2} e^{\pi i \sigma\left(D^{\prime}\right) / 4} \sum_{P \subset D \backslash I}(-1)^{|P|+|P|_{+}} \frac{i^{\# P}+i^{\#(P \cup I)}}{1-i} .
\end{aligned}
$$

There exists a natural correspondence between the subdiagrams of $D^{\prime}$ and $D \backslash I$. If $P^{\prime}$ and $P$ are the corresponding subdiagrams, then (by Figures 4 (c)-(d)), $|P|=\left|P^{\prime}\right|$, $|P|_{+}=\left|P^{\prime}\right|_{+}, \# P=\# P^{\prime}-n^{2}, \#(P \cup I)=\#\left(P^{\prime}\right)-n^{2}+2 n+1$, where $n \geq 0$ is the number of components in the part of $P$ corresponding to the part of $D$ shown in Figure 4 (a). Since $i^{-n^{2}}-i^{-n^{2}+2 n+1}=1-i$, it follows that $R\left(D^{\prime}\right)=R(D)$.

LEMMA 2.2. $W(D)$ remains unchanged under the first Kirby move in Figure 5.

PROOF. Clearly, $b_{ \pm}\left(D \cup U_{ \pm}\right)=b_{ \pm}(D)+1, b_{ \pm}\left(D \cup U_{\mp}\right)=b_{ \pm}(D)$, and

$$
f_{D \cup U_{ \pm}}(\omega, \ldots, \omega)=f_{D}(\omega, \ldots, \omega) \cdot f_{U_{ \pm}}(\omega) .
$$

Hence $W\left(D \cup U_{-}\right)=W(D)=W\left(D \cup U_{+}\right)$.

LEMMA 2.3. $W(E)$ remains unchanged under the Fenn-Rourke moves of the diagram $E$ in Figures 4 (a)-(b) if for arbitrary diagrams $D$ and $D^{\prime}$ that differ as in Figures 3 (a)-(b) the following equality holds

$$
f_{D}(\omega, \alpha, \alpha, \ldots, \alpha)=f_{D^{\prime}}(\omega, \alpha, \alpha, \ldots, \alpha) .
$$

Proof. Clearly, the Fenn-Rourke moves in Figures 4 (a)-(b) are decomposed into $l$ second Kirby moves in Figures 3 (a)-(b) (for $m=l, \ldots, 1$ ) respectively, and one first Kirby move in Figure 5. Thus it follows from Lemma 2.1 and Lemma 2.2 that we only need to check the equality $f_{D}(\omega, \ldots, \omega)=f_{D^{\prime}}(\omega, \ldots, \omega)$, where $D$ and $D^{\prime}$ are shown in Figure 3 (a) or 3 (b) and their first two components are specified. Let $n=|D|=\left|D^{\prime}\right|$ and $k_{2}, k_{3}, \ldots, k_{n} \geq 0$ be arbitrary integers. It suffices to verify that

$$
f_{D}\left(\omega, \alpha^{k_{2}}, \alpha^{k_{3}}, \ldots, \alpha^{k_{n}}\right)=f_{D^{\prime}}\left(\omega, \alpha^{k_{2}}, \alpha^{k_{3}}, \ldots, \alpha^{k_{n}}\right) .
$$



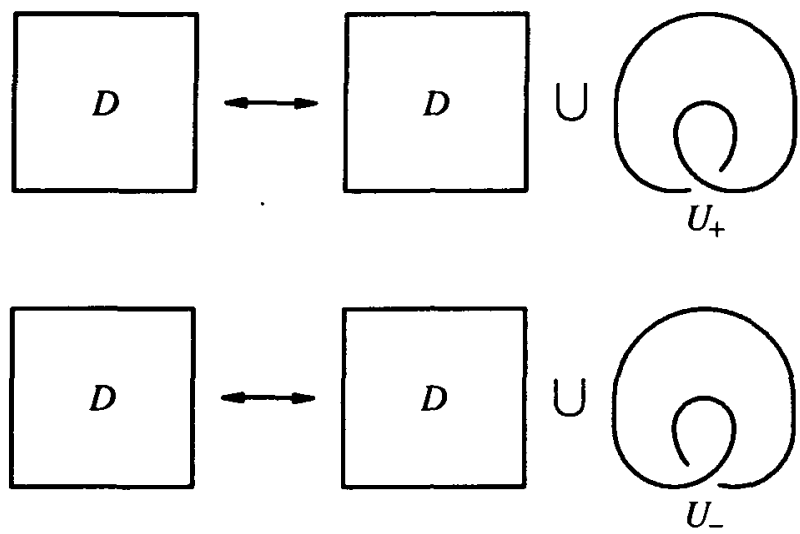

FIGURE 5 .

This equality is clear for $k_{2}=0$. If $k_{i}=0$, for some $i \geq 3$, we may consider $D \backslash D_{i}$ and $D^{\prime} \backslash D^{\prime}{ }_{i}$ instead of $D$ and $D^{\prime}$. Therefore we may assume that $k_{3}, \ldots, k_{n} \neq 0$. Let $C$ and $C^{\prime}$ be the diagrams obtained from $D$ and $D^{\prime}$ by taking $k_{i}$ curves, for each $i \geq 3$, close and parallel to the $i$-th component. Considering $C$ and $C^{\prime}$ instead of $D$ and $D^{\prime}$ we may assume that $k_{3}, \ldots, k_{n}=1$. By induction on $k_{2}$ it follows that the above equality for $k_{2}=1$ implies the analogous equation for arbitrary $k_{2}$. Indeed, suppose that $k_{2} \geq 2$. Let $K=D^{\prime(1,2,1, \ldots .1)}$ with $|K|=n+1$ and $J^{\prime}$ be the second component of $D^{\prime}$. Obviously, we have $D^{\prime\left(k_{1}, k_{2}, 1 \ldots, 1\right)}=K^{\left(k_{1}, k_{2}-1,1,1 \ldots, 1\right)}$. The induction hypothesis for diagrams $K$ and $D \cup J^{\prime}$ then gives that

$$
\begin{aligned}
f_{D^{\prime}}\left(\omega, \alpha^{k_{2}}, \alpha, \ldots, \alpha\right) & =f_{K}\left(\omega, \alpha^{k_{2}-1}, \alpha, \alpha, \ldots, \alpha\right) \\
& =f_{D \cup J^{\prime}}\left(\omega, \alpha^{k_{2}-1}, \alpha, \alpha, \ldots, \alpha\right) \\
& =f_{D}\left(\omega, \alpha^{k_{2}}, \alpha, \ldots, \alpha\right) .
\end{aligned}
$$

PROOF OF THEOREM 1.3 FOR $r=4$ AND $k=1$. From now on assume that the Kauffman bracket is calculated at $a=e^{\pi i / 8}$. We prove the invariance of $W(D)$ under the move in Figure 4 (a). The invariance under the move in Figure 4 (b) is verified analogously. Let $D$ and $D^{\prime}$ be the diagrams shown in Figure 3 (a). By $I$ and $I$ ' we denote their first components.

Since $\omega=\alpha^{2}-\sqrt{2} \alpha$ it follows by Lemma 2.3 that we must only show that

$$
\langle D \circ I\rangle-\sqrt{2}\langle D\rangle=\left\langle D^{\prime} \circ I^{\prime}\right\rangle-\sqrt{2}\left\langle D^{\prime}\right\rangle .
$$

Applying (a) to the crossings marked in Figure $6(a)$, we obtain $-\sqrt{2}\langle D\rangle=\sqrt{2} a^{3}\langle S\rangle$, $\langle D \circ I\rangle=2\langle Q\rangle-\langle T\rangle,-\sqrt{2}\left\langle D^{\prime}\right\rangle=-\sqrt{2}\left\langle S^{\prime}\right\rangle$ and $\left\langle D^{\prime} \circ I^{\prime}\right\rangle=-2 a^{-3}\left\langle Q^{\prime}\right\rangle+a^{-3}\left\langle T^{\prime}\right\rangle$. 

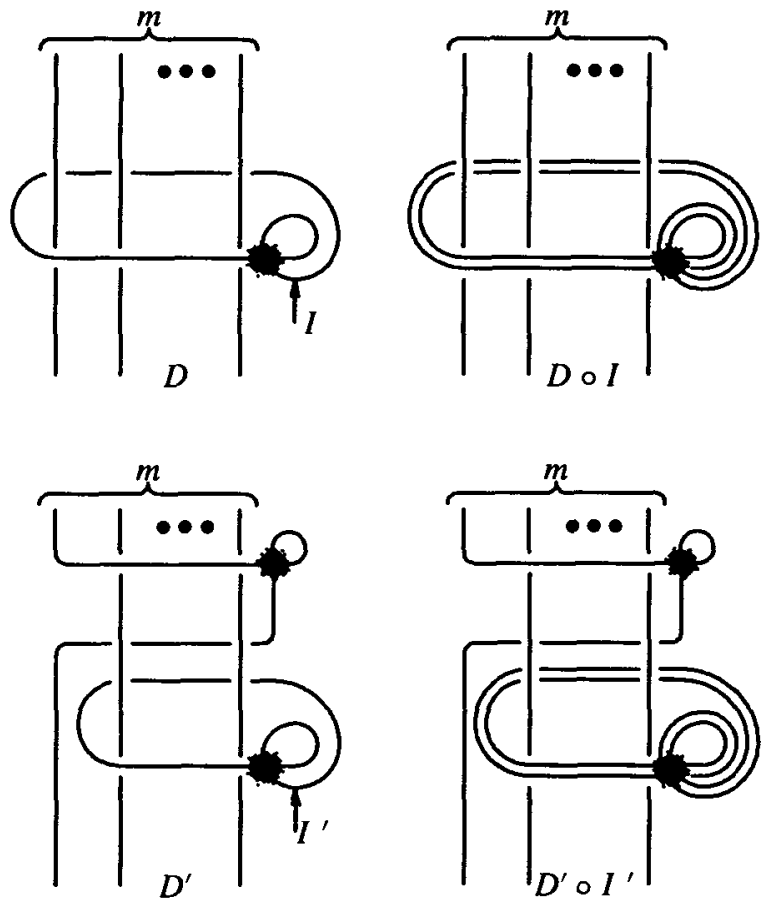

(a)
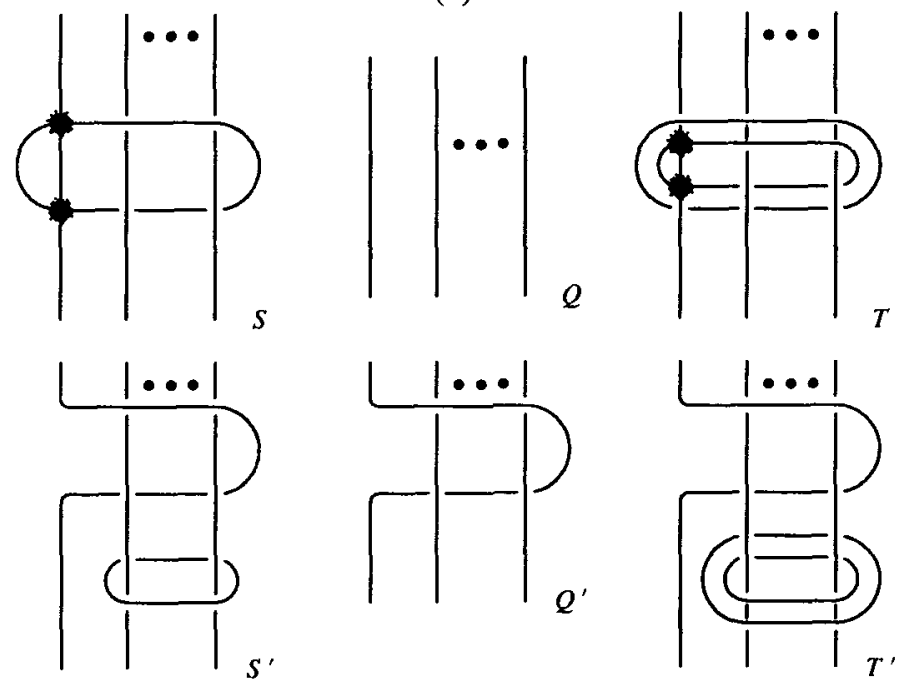

(b)

FIGURE 6. 


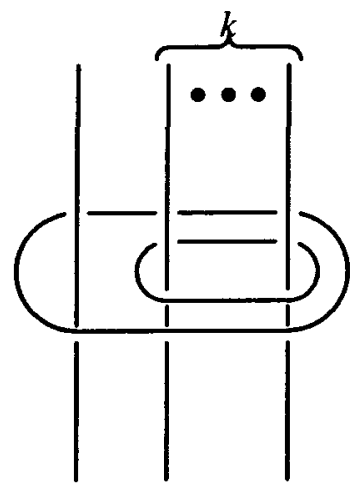

(a)

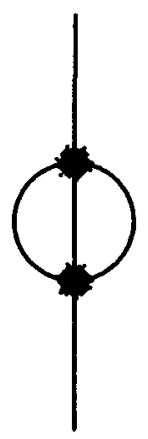

(b)

FIGURE 7.
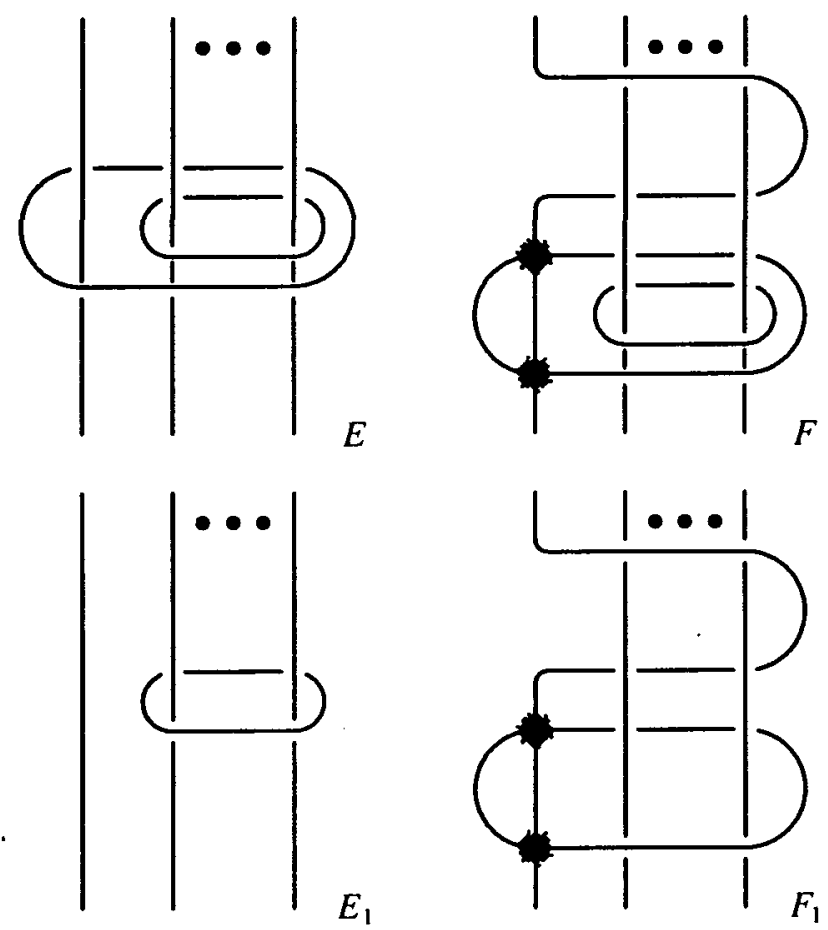

FIGURE 8. 
To complete the proof of Theorem 1.3 for $r=4$ and $k=1$ we need the following simple lemma.

LEMMA 2.4. Suppose that the diagram A contains the part shown in Figure 7 (a), where $k \geq 0$. Then $\langle A\rangle=0$.

PROOF. By property (a) of the Kauffman bracket, we may assume that $A$ has no crossings outside the part shown. It is easy to see that $A$ contains the part shown in Figure 7 (b). Applying (a) to the two marked crossings in Figure 7 (b) and using (b) one can easily obtain that $\langle A\rangle=0$.

Applying (a) to the crossings of $T$ and $F_{1}$ marked in Figure 6 (b) and Figure 8, using Proposition 1.2 (for the first and the last equalities) and Lemma 2.4 (for the second equality) we get that

$$
\langle T\rangle=(1+i)\left\langle F_{1}\right\rangle+\frac{1+i}{\sqrt{2}}\langle E\rangle=(1+i)\left\langle F_{1}\right\rangle=2\langle Q\rangle+\sqrt{2}\left\langle S^{\prime}\right\rangle
$$

Hence, $\langle D \circ I\rangle-\sqrt{2}\langle D\rangle=\sqrt{2} a^{3}\langle S\rangle-\sqrt{2}\left\langle S^{\prime}\right\rangle$. Clearly, $(* *)$ is equivalent to the equality $\sqrt{2} a^{3}\langle S\rangle=-2 a^{-3}\left\langle Q^{\prime}\right\rangle+a^{-3}\left\langle T^{\prime}\right\rangle$. Using Lemma 2.4 (for first equality), applying (a) to the crossings of $F$ and $S$ marked in Figure $6(\mathrm{~b})$ and Figure 8 and using Proposition 1.2 (for the last two equalities) we obtain that

$$
\begin{aligned}
\sqrt{2} a^{3}\langle S\rangle & =\sqrt{2} a^{3}\langle S\rangle+a^{-1}\langle F\rangle \\
& =\sqrt{2} a^{3}\langle S\rangle+a^{-3}\left\langle T^{\prime}\right\rangle+\sqrt{2} a^{-3}\left\langle E_{1}\right\rangle \\
& =-2 a^{-3}\left\langle Q^{\prime}\right\rangle+a^{-3}\left\langle T^{\prime}\right\rangle
\end{aligned}
$$

and we are done.

\section{Acknowledgements}

The authors would like to thank W. B. R. Lickorish and A. B. Skopenkov for useful discussions.

\section{References}

[1] R. P. Fenn and C. P. Rourke, 'On Kirby's calculus of links', Topology 18 (1979), 1-15.

[2] L. H. Kauffman, 'State models and the Jones polynomial', Topology 26 (1987), 395-407.

[3] R. Kirby, 'A calculus for framed links in $S^{3}$, Invent. Math. (1) 45 (1978), 35-56.

[4] R. Kirby and P. Melvin, 'The 3-manifolds invariants', Invent. Math. 105 (1991), 473-545. 
[5] W. B. R. Lickorish, 'Polynomials for links', Bull. London Math. Soc. 20 (1988), 558-588.

[6] —_. 'The skein method for three-manifold invariants', Pacific J. Math. 149 (1991), 337-347.

[7] - 'The Skein method for three-manifold invariants', J. Knot Theory Ramifications 2 (1993), 171-194.

[8] V. Prasolov and A. Sossinsky, Knots, links, braids and 3-manifolds, Transl. Math. Monographs 154 (Amer. Math. Soc., Providence, 1997).

[9] N. Saveliev, Lectures on the topology of 3-manifolds (Walter de Gruyter, Berlin, 1999).

Department of Differential Geometry

Faculty of Mechanics and Mathematics

Moscow State University

Moscow 119899

Russia

e-mail: rafikov@mccme.ru
Institute for Mathematics, Physics and Mechanics University of Ljubljana P.O. Box 2964 1001 Ljubljana Slovenia e-mail: dusan.repovs@fmf.uni-lj.si

Department of Mathematics

University of Modena and Reggio Emilia

Via Campi 213/B

41100 Modena

Italy

e-mail: spaggiari@unimo.it 